${ }^{1}$ Dept of Respiratory Medicine and Allergy, Tosei General Hospital, Seto, Aichi, Japan. ${ }^{2}$ Dept of Pathology, Tosei General Hospital, Seto, Aichi, Japan. ${ }^{3}$ Dept of Pathology, Nagasaki University Graduate School of Biomedical Sciences, Nagasaki, Japan.

Correspondence: Yasuhiro Kondoh, Dept of Respiratory Medicine and Allergy, Tosei General Hospital 160 Nishioiwake-cho, Seto, Aichi 489-8642, Japan. E-mail: lung@tosei.or.jp

Received: July 212014 | Accepted after revision: Nov 152014 | First published online: Feb 052015

Conflict of interest: None declared.

\title{
References
}

$1 \quad$ Stone JH, Zen Y, Deshpande V. IgG4-related disease. N Engl J Med 2012; 366: 539-551.

2 Hamano H, Kawa S, Horiuchi A, et al. High serum IgG4 concentrations in patients with sclerosing pancreatitis. N Engl J Med 2001; 344: 732-738.

3 Ryu JH, Sekiguchi H, Yi ES. Pulmonary manifestations of immunoglobulin G4-related sclerosing disease. Eur Respir J 2012; 39: 180-186.

4 Watanabe N, Sakamoto K, Taniguchi $\mathrm{H}$, et al. Efficacy of combined therapy with cyclosporin and low-dose prednisolone in interstitial pneumonia associated with connective tissue disease. Respiration 2014; 87: 469-477.

5 Umehara H, Okazaki K, Masaki Y, et al. Comprehensive diagnostic criteria for IgG4-related disease (IgG4-RD), 2011. Mod Rheumatol 2012; 22: 21-30.

6 Inoue $\mathrm{D}$, Zen $\mathrm{Y}, \mathrm{Abo} \mathrm{H}$, et al. Immunoglobulin G4-related lung disease: CT findings with pathologic correlations. Radiology 2009; 251: 260-270.

7 Zen Y, Inoue D, Kitao A, et al. IgG4-related lung and pleural disease: a clinicopathologic study of 21 cases. Am J Surg Pathol 2009; 33: 1886-1893.

8 Matsui S, Hebisawa A, Sakai F, et al. Immunoglobulin G4-related lung disease: clinicoradiological and pathological features. Respirology 2013; 18: 480-487.

9 Ito $\mathrm{M}$, Yasuo $\mathrm{M}$, Yamamoto $\mathrm{H}$, et al. Central airway stenosis in a patient with autoimmune pancreatitis. Eur Respir J 2009; 33: 680-683.

10 Pifferi M, Cicco MD, Bush A, et al. Uncommon pulmonary presentation of IgG4-related disease in a 15-year-old boy. Chest 2013; 144: 669-671.

11 Matsui S, Sugiyama E, Taki H, et al. A case of bronchiolitis with IgG4-positive plasma cells infiltration associated with Mikulicz's disease and autoimmune pancreatitis. Nihon Kokyuuki Gakkai Zasshi 2009; 47: 139-144.

12 Hasegawa $\mathrm{H}$, Nakamura $\mathrm{Y}$, Kono $\mathrm{M}$, et al. Bronchiolitis with IgG4-positive plasma cell infiltration in a patient with bronchial asthma. Nihon Kokyuuki Gakkai Zasshi 2010; 48: 317-321.

13 Ishida M, Miyamura T, Sato S, et al. Pulmonary arterial hypertension associated with IgG4-related disease. Intern Med 2014; 53: 493-497.

14 Montani D, Price LC, Dorfmuller P, et al. Pulmonary veno-occlusive disease. Eur Respir J 2009; 33: 189-200.

15 Sherrick AD, Brown LR, Harms GF, et al. The radiographic findings of fibrosing mediastinitis. Chest 1994; 106: 484-489.

\section{Cystic fibrosis bone disease: is the CFTR corrector C18 an option for therapy?}

\author{
To the Editor:
}

Mutations in the gene encoding the chloride ion channel CFTR (cystic fibrosis transmembrane conductance regulator) result in cystic fibrosis (CF), the most common lethal autosomal recessive genetic disease, which causes a number of long-term health problems, such as bone disease. Osteoporosis and increased vertebral fracture risk associated with CF disease are becoming more important as the life expectancy of patients continues to improve. The aetiology of low bone density is multifactorial, and is most probably a combination of inadequate peak bone mass during puberty and increased bone loss in adults [1]. Body mass index, male sex, advanced pulmonary disease, malnutrition and chronic therapies are established additional risk factors for CF-related bone disease. In multiple studies with a large cohort of adolescent and adult CF patients, the incidence of osteopenia and osteoporosis ranges from 34\% to $79 \%$ $[1,2]$. This further translated to a 100 -fold greater risk of vertebral compression, which can decrease lung function, thus accelerating the course of the disease and decreasing the patient's quality of life.

Emerging data suggest a direct genetic component to the development of CF-related bone disease. F508del is the most common CFTR mutation, with $>80 \%$ of patients being at least heterozygous [3]. We recently 
discovered a defective CFTR-mediated chloride channel activity and severe deficit of the release of osteoprotegerin (OPG) in primary osteoblasts (cells that form bone) obtained from a 25 -year-old CF male with F508del/G542X mutation in CFTR [4]. Recent evidence from clinical and CF model studies suggests that loss of functional CFTR not only causes osteoblast dysfunction in bones $[5,6]$ but also in airway cartilage, which may be important for airway development and dysfunction. Reports have shown that CF mice and pigs, but not epithelial sodium channel-overexpressing mice with CF-like lung disease, show abnormalities in tracheal cartilage development with early airflow obstruction [7-10], suggesting a critical role of CFTR dysfunction independent of deficient ion transport/airway surface liquid depletion. From rib explants harvested during lung transplantation in adolescents with $\mathrm{CF}$, we validated the genetic
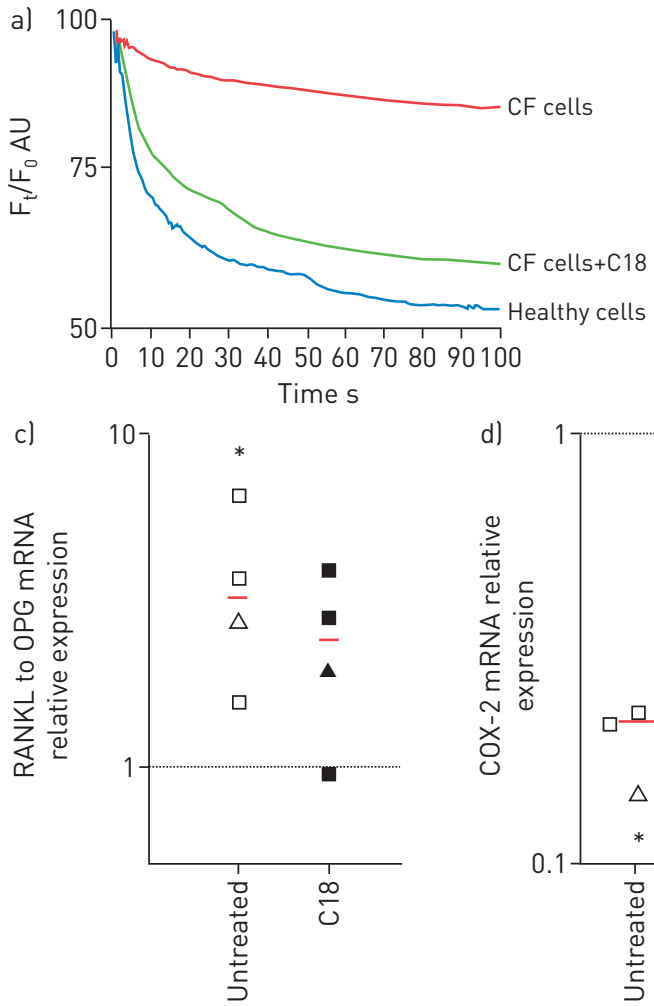
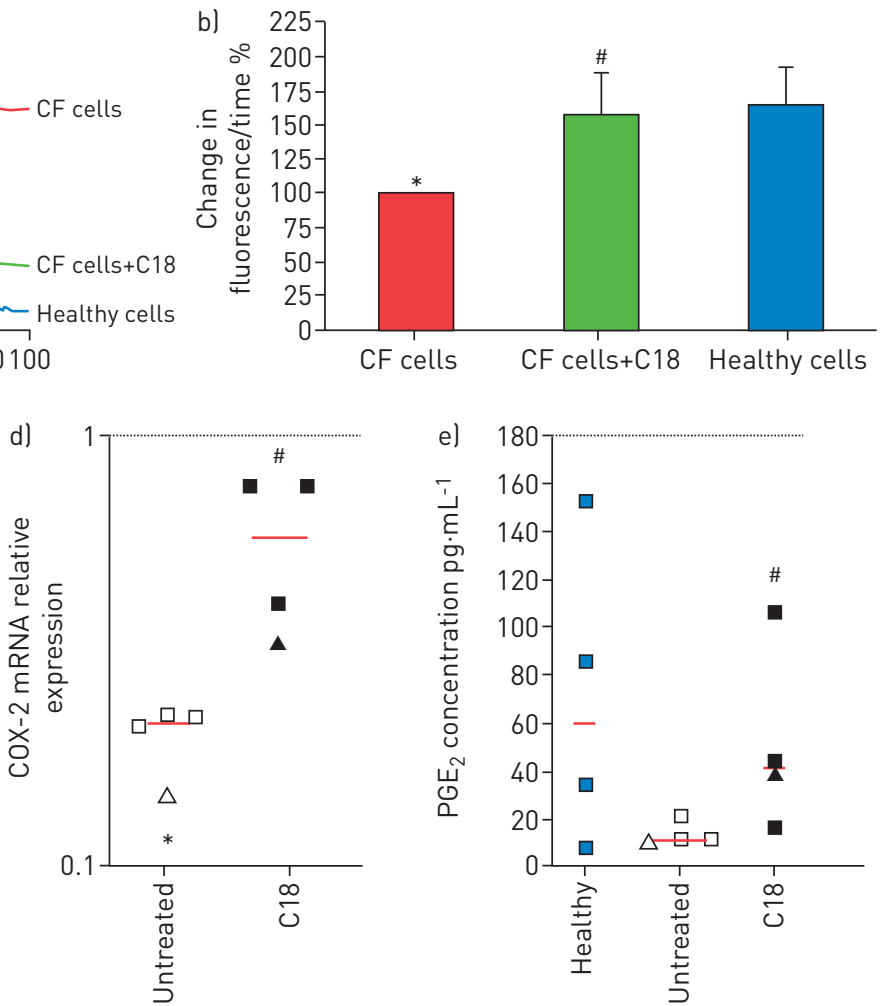

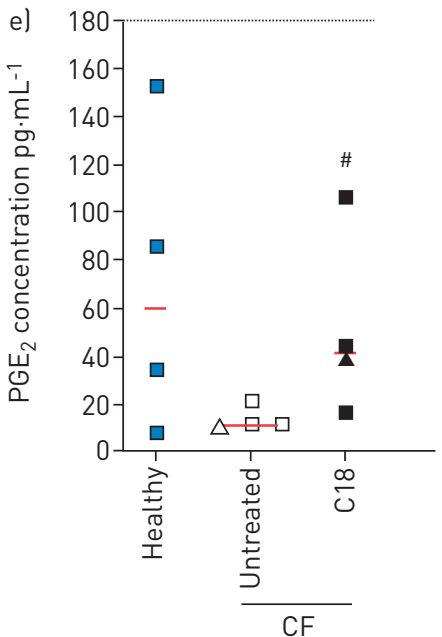

FIGURE 1 The cystic fibrosis transmembrane conductance regulator (CFTR) corrector C18 restores CFTR-dependent chloride transport in F508del osteoblasts. a) Representative traces and b) quantification of F508del-CFTR channel activity assessed with iodide efflux in F508del osteoblasts (cystic fibrosis (CF) cells pretreated with either $10 \mu \mathrm{M} \mathrm{C18}$ or dimethylsulfoxide (DMSO) for $24 \mathrm{~h}$ ). CFTR-dependent chloride transport was assessed in osteoblasts by iodine quenching of halide-sensitive yellow fluorescent protein (YFP)-H148Q/I152 L protein (Life Technologies, Saint Aubin, France). The probe was transfected into the cells and, after $48 \mathrm{~h}$ culture, conductance was stimulated for CFTR channel activity with a mixture composed of forskolin, 3-isobutyl-1-methylxanthine and apigenin (each component at a concentration of $10 \mu \mathrm{M})$. Iodine solution $(140 \mathrm{mM})$ was then added and fluorescence was recorded continuously using a plate reader ( $400 \mathrm{~ms}$ per point; $F_{t}$ ) for a 250 -s period (baseline; $F_{0}$ ). The decrease in the percentage of $F_{t} / F_{0}$ represents the increase of CFTR chloride conductance. The specific CFTR inhibitor CFTR $_{\text {inh-172 }}(10 \mu \mathrm{M}$ for $24 \mathrm{~h})$ was used to verify that the signal observed was CFTR-mediated in healthy osteoblasts (non-CF), as we previously showed [11]. In

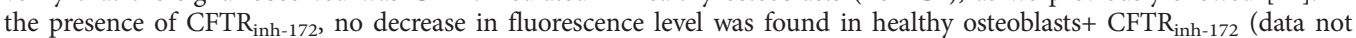
shown) [11]. The initial iodine influx rate following the addition of each solution was computed from changes in YFP fluorescence using nonlinear regression. For quantitative analysis, the slope of fluorescence quenching was calculated using a linear regression and correlated to the level of the chloride conductance (iodine uptake). The rate of change in fluorescence over time in F508del osteoblasts ( $\mathrm{n}=4$ CF cells pretreated with either $10 \mu \mathrm{M}$ C18 or DMSO for $24 \mathrm{~h}$ ) is presented in the bar graph. The CFTR corrector C18 ameliorates the RANK ligand (RANKL)/osteoprotegerin (OPG) mRNA ratio and increases both the cyclo-oxygenase (COX)-2 mRNA expression and prostaglandin (PG) $\mathrm{E}_{2}$ production in F508del osteoblasts. c) RANKL/OPG mRNA relative expression in F508del osteoblasts compared with healthy osteoblasts, treated or not with C18. d) Relative expression of COX-2 mRNA in F508del osteoblasts compared to healthy osteoblasts, treated or not with C18. e) Basal production of COX-2 metabolite $\mathrm{PGE}_{2}$ assessed by enzyme immunosorbent assay in 24-h supernatants. All experiments were carried out after a 14-day culture-confluent period in healthy and CF osteoblasts ( $n=4$ healthy donors, $n=3$ CF patients homozygous for the F508del mutation; $n=1$ CF patient heterozygous for the F508del mutation in CFTR), treated or not with C18 (10 $\mu \mathrm{M}$ for $24 \mathrm{~h})$. Real-time PCR data were calculated following the $2^{-\Delta \Delta \mathrm{Ct}}$ method. Each square or triangle represents one donor value; the red bar represents the median value. ${ }^{*}: \mathrm{p}<0.05$ versus healthy osteoblasts; ${ }^{*}: \mathrm{p}<0.05$ versus untreated F508del osteoblasts. 
contribution by which F508del mutation in CFTR resulted in a severe, defective osteoblast maturation arising from an increased RANK ligand (RANKL)/OPG mRNA ratio and a drastic reduction in production of the cyclo-oxygenase (COX)-2 metabolite prostaglandin ( $\mathrm{PG}$ ) $\mathrm{E}_{2}$, a key regulator of bone turnover [11]. Another report also showed an elevated RANKL/OPG ratio in the serum of children and adolescents with CF compared with that observed in healthy controls [2].

New treatments that target the F508del mutation through the use of potentiators and correctors of chloride channels are being developed in the care of CF-related lung pathology. Certain small molecules defined as "dual-acting potentiator-correctors" with both activities have been shown to partially rescue the functional expression of F508del-CFTR on the membrane of epithelia in patient-derived airway cultures, providing the rationale for clinical trials of the best compounds, including VX-809 [12, 13]. Recent in vitro studies have shed considerable light on the potential mechanism of action of the structurally related compounds VX-809 and C18 (also known as VRT-534), showing these correctors bind to full-length F508del-CFTR and enhance the channel-active form of the metastable F508del-CFTR protein after its biosynthetic rescue [12]. We therefore tested the effect of the corrector C18 on CFTR channel activity, the expression level of RANKL/OPG mRNA ratio and COX-2/PGE 2 expression and production in osteoblasts with the F508del mutation. F508del osteoblasts were obtained from trabecular bone explants prepared from rib fragments harvested during lung transplantation, as previously described [11]. Normal osteoblasts, used as controls, were obtained from fresh trabecular bone explants of healthy young adults who underwent trauma surgery. The bone samples were obtained with informed patient consent after approval by the local research ethics committee.

First, we examined the efficacy of $\mathrm{C} 18$ in enhancing F508del-CFTR chloride function in primary osteoblast cell cultures obtained from four different adolescents with CF (three young patients: two 13- and 15-year-old females and a 14-year-old male homozygous for the F508del CFTR mutation, and a 14-year-old male with the heterozygous F508del/G542X mutation in CFTR). C18 was found to greatly enhance F508del-CFTR channel function in all F508del osteoblast cultures. The extent of functional rescue caused by $\mathrm{C} 18$ treatment was $\sim 85 \%$ of the mean CFTR function measured in healthy osteoblasts as reported in figure 1a and $\mathrm{b}$. We found a similar functional rescue in F508del osteoblasts when treated with the small molecule VX-809, a structurally related corrector compound in clinical trials (data not shown).

Secondly, real-time PCR showed that F508del osteoblasts treated with C18 have a $34 \%$ reduction of RANKL/OPG mRNA ratio compared with untreated F508del osteoblasts in which RANKL/OPG ratio was higher as compared with that found in normal osteoblasts (fig. 1c). Interestingly, we also found that treatment with $\mathrm{C} 18$ resulted in a significant increase of COX-2 expression and $\mathrm{PGE}_{2}$ production in F508del osteoblasts (fig. 1d and e). Prior reports have shown that COX-2 activity and $\mathrm{PGE}_{2}$ production are required for a full activation of $\mathrm{Wnt} / \beta$-catenin signalling pathway in osteoblasts that is critically involved in the regulation of skeletal growth and efficient bone fracture healing. Moreover, osteoblast differentiation and maturation of bone marrow stem cells is deficient in the absence of COX-2, and this can be compensated for by the addition of $\mathrm{PGE}_{2}[14]$.

Clinical trials are underway with the goal of finding new potential treatments that might prevent the development of CF-related bone disease, including antiresorptive agents such as oral bisphosphonates, and anabolic agents such as human recombinant growth hormones and parathyroid hormone [1]. A recent clinical study provides convincing evidence that the oral bisphosphonate alendronate is effective, well tolerated and safe for young patients with CF [15]. However, the use of bisphosphonates in children with $\mathrm{CF}$ is controversial because of potential long-term safety concerns including oversuppression of bone formation. The European CF bone mineralisation guidelines recently highlighted controversial issues, such as the use of bisphosphonates and vitamin $\mathrm{D}$ supplementation regimens in children and adolescents, indicating the need for other therapeutic trials for treating CF-related skeletal deficits [1]. Thus, there is an urgent need for an efficient and safe antiosteopenic treatment to ameliorate osteoblast activity and, thus, favour the bone formation in patients with CF.

Therefore, the discovery of CFTR modulators acting as a "dual" F508del-CFTR correctors and potentiators, such as $\mathrm{C} 18$, leading to an increase of $\mathrm{COX}-2 / \mathrm{PGE}_{2}$ expression and production, and counteracting an elevated RANKL/OPG ratio in osteoblasts with the F508del mutation, represents a step forward in the development of potential new therapies to treat bone disease in patients with CF bearing the F508del mutation. 
Frédéric Velard ${ }^{1}$, Martial Delion ${ }^{1}$, Flora Lemaire ${ }^{1}$, Olivier Tabary ${ }^{2}$, Christine Guillaume $^{1}$, Françoise Le Pimpec Barthès ${ }^{3}$, Lhousseine Touqui ${ }^{4}$, Sophie Gangloff ${ }^{1}$, Isabelle Sermet-Gaudelus ${ }^{5}$ and Jacky Jacquot $^{1}$

${ }^{1}$ EA 4691 «Biomatériaux et Inflammation en site osseux», SFR CAP-Santé (FED 4231), Université Reims ChampagneArdenne, Reims, France. ${ }^{2}$ Inserm U938, Hospital St-Antoine, Paris, France. ${ }^{3}$ Département de Chirurgie Thoracique, Hôpital Européen Georges Pompidou, Paris, France. ${ }^{4}$ Inserm U874, Unité de Défense Innée et Inflammation, Institut Pasteur, Paris, France. ${ }^{5}$ Unité de Pneumo-Pédiatrie Allergologie, Hôpital Necker, Inserm U1551, Université Paris Sorbonne, Paris, France.

Correspondence: Jacky Jacquot, EA 4691, BIOS, SFR CAP-Santé (FED 4231), Université Reims Champagne Ardenne, 1, Avenue du Maréchal Juin, 51095, Reims, France. E-mail: jacky.jacquot@inserm.fr

Received: June 262014 | Accepted after revision: Oct 272014 | First published online: Nov 272014

Support statement: This study was supported, in part, by funds from the French Cystic Fibrosis Association Vaincre la Mucoviscidose (grant RF20120600625 to J. Jacquot). M. Delion is a PhD scholarship recipient from the Ministère de l'Enseignement Supérieur et de la Recherche, Paris, France. C18 was provided by Vertex Pharmaceuticals.

Conflict of interest: None declared.

Acknowledgements: The authors thank all children who participated in this study. We also acknowledge the provision of CFTR corrector VRT-534/C18 by Vertex Pharmaceuticals (Boston, MA, USA).

\section{References}

1 Sermet-Gaudelus I, Bianchi ML, Garabedian M, et al. European cystic fibrosis bone mineralisation guidelines. J Cyst Fibros 2011; 10: Suppl. 2, S16-S23.

2 Ambroszkiewicz J, Sands D, Gajewska J, et al. Bone turnover markers, osteoprotegerin and RANKL cytokines in children with cystic fibrosis. Adv Med Sci 2013; 58: 338-343.

3 Castellani C, Cuppens H, Macek M Jr, et al. Consensus on the use and interpretation of cystic fibrosis mutation analysis in clinical practice. J Cyst Fibros 2008; 7: 179-196.

4 Gimenez-Maitre A, Le Henaff C, Norez C, et al. Deficit of osteoprotegerin release by osteoblasts from a patient with cystic fibrosis. Eur Respir J 2012; 39: 780-781.

5 King SJ, Topliss DJ, Kotsimbos T, et al. Reduced bone density in cystic fibrosis: $\Delta \mathrm{F} 508$ mutation is an independent risk factor. Eur Respir J 2005; 25: 54-61.

6 Le Henaff C, Hay E, Velard F, et al. Enhanced F508del-CFTR channel activity ameliorates bone pathology in murine cystic fibrosis. Am J Pathol 2014; 184: 1132-1141.

7 Bonora M, Riffault L, Marie S, et al. Morphological analysis of the trachea and pattern of breathing in $\beta \mathrm{ENaC}-\mathrm{Tg}$ mice. Respir Physiol Neurobiol 2011; 178: 346-348.

8 Bonvin E, Le Rouzic P, Bernaudin JF, et al. Congenital tracheal malformation in cystic fibrosis transmembrane conductance regulator-deficient mice. J Physiol 2008; 586: 3231-3243.

9 Meyerholz DK, Stoltz DA, Namati E, et al. Loss of cystic fibrosis transmembrane conductance regulator function produces abnormalities in tracheal development in neonatal pigs and young children. Am J Respir Crit Care Med 2010; 182: 1251-1261.

10 Adam RJ, Michalski AS, Bauer C, et al. Air trapping and airflow obstruction in newborn cystic fibrosis piglets. Am J Respir Crit Care Med 2013; 188: 1434-1441.

11 Velard F, Delion M, Le Henaff C, et al. Cystic fibrosis and bone disease: defective osteoblast maturation with the F508del mutation in cystic fibrosis transmembrane conductance regulator. Am J Respir Crit Care Med 2014; 189: 746-748.

12 Eckford PD, Ramjeesingh M, Molinski S, et al. VX-809 and related corrector compounds exhibit secondary activity stabilizing active F508del-CFTR after its partial rescue to the cell surface. Chem Biol 2014; 21: 666-678.

13 Van Goor F, Hadida S, Grootenhuis PD, et al. Correction of the F508del-CFTR protein processing defect in vitro by the investigational drug VX-809. Proc Natl Acad Sci USA 2011; 108: 18843-18848.

14 Blackwell KA, Raisz LG, Pilbeam CC. Prostaglandins in bone: bad cop, good cop? Trends Endocrinol Metab 2010; 21: 294-301.

15 Bianchi ML, Colombo C, Assael BM, et al. Treatment of low bone density in young people with cystic fibrosis: a multicentre, prospective, open-label observational study of calcium and calcifediol followed by a randomised placebo-controlled trial of alendronate. Lancet Respir Med 2013; 1: 377-385. 